\title{
Extraction of Substrate Parameters for RF MOSFETs Based on Four-Port Measurement
}

\author{
Shih-Dao Wu, Guo-Wei Huang, Member, IEEE, Kun-Ming Chen, Member, IEEE, Chun-Yen Chang, Fellow, IEEE, \\ Hua-Chou Tseng, and Tsun-Lai Hsu
}

\begin{abstract}
In this work, a new method for extracting substrate parameters of radio frequency (RF) metal oxide semiconductor field effect transistors (MOSFETs) based on four-port measurement is presented. A T-liked substrate resistance network is used and the values of all components in the cold MOSFETs were extracted directly from the four-port data between $250 \mathrm{MHz}$ and 8.5 GHz. The output admittance $Y_{22}$ can be well modeled up to 26.5 GHZ based on the extracted substrate resistances and the other extrinsic capacitances extracted from an active device.
\end{abstract}

Index Terms-Four-port measurement, radio frequency (RF) metal oxide semiconductor field effect transistors (MOSFETs), substrate resistance.

\section{INTRODUCTION}

I T IS known that the substrate resistances have significant effect on output characteristics of radio frequency (RF) metal oxide semiconductor field effect transistors (MOSFETs) and can't be ignored. Recently, several extraction methods of substrate resistances have been reported [1]-[5]. However, the substrate resistances were simplified to a single substrate resistance [1]-[3] or were calculated by process parameters [4], earlier reports even optimized substrate resistances by curve fitting techniques [6]. It is due to that RF MOSFETs were always measured in conventional two-port common source configuration. As the source and substrate terminals are connected together, each resistance components in the entire substrate resistances network, such as resistances from source and drain to body, and body series resistance itself, will not be able to separate according to the insufficient information provided by two-port measurement data. In this work, an extraction method based on four-port measurement is proposed. All the components of the substrate network including junction capacitances and substrate resistances can be directly extracted from measured raw data.

\section{Four-Port Measurement AND Extraction Method}

In this study, the layout of the RF MOSFET is designed as gate, drain, source and body were connected individually to four signal pads. These four signal pads incorporated with a reference ground form a four-port ground-signal-ground (GSG) test

Manuscript received November 16, 2004; revised March 29, 2005. The review of this letter was arranged by Associate Editor A. Weisshaar.

S. D. Wu and C.-Y. Chang are with the Institute of Electronics, National Chiao Tung University, Hsinchu, Taiwan, R.O.C.

G.-W. Huang and K.-M. Chen are with the National Nano Device Laboratories, Hsinchu, Taiwan, R.O.C. (e-mail: gwhuang@ndl.gov.tw).

H.-C. Tseng and T.-L. Hsu are with the United Microelectronics Corporation, Hsinchu, Taiwan, R.O.C

Digital Object Identifier 10.1109/LMWC.2005.850566 structure and the RF MOSFET can be treated as a four-port device and characterized by four-port measurement [7]. Fig. 1 shows a small-signal equivalent circuit of a four-port MOSFET, in which $g_{\mathrm{m}}$ represents the transconductance and $R_{\mathrm{ch}}$ is the channel resistance. $C_{\mathrm{gs}}$ and $C_{\mathrm{gd}}$ represent the gate to source and gate to drain capacitances, respectively. $C_{\mathrm{gb}}$ is the capacitance between gate and body. $C_{\mathrm{sb}}$ and $C_{\mathrm{db}}$ represent the junction capacitance of source and drain, respectively. There is also a T-liked substrate resistance network consists of three resistances $R_{\mathrm{sb}}, R_{\mathrm{db}}$, and $R_{\mathrm{bb}}$, which represent resistances from source and drain to body, and body series resistance, respectively.

A $4 \times 4 Y$-parameter matrix shown in (1) which represents the DUT can be obtained by four-port measurement and proper pad de-embedding.

$$
\left[\begin{array}{c}
i_{g} \\
i_{\mathrm{D}} \\
i_{S} \\
i_{B}
\end{array}\right]=\left[\begin{array}{cccc}
Y_{\mathrm{GG}} & Y_{G D} & Y_{G S} & Y_{G B} \\
Y_{\mathrm{DG}} & Y_{\mathrm{DD}} & Y_{D S} & Y_{D B} \\
Y_{\mathrm{SG}} & Y_{\mathrm{SD}} & Y_{\mathrm{SS}} & Y_{S B} \\
Y_{B G} & Y_{\mathrm{BD}} & Y_{\mathrm{BS}} & Y_{\mathrm{BB}}
\end{array}\right]\left[\begin{array}{c}
v_{g} \\
v_{\mathrm{D}} \\
v_{S} \\
v_{B}
\end{array}\right] .
$$

While the bias voltage on each terminal is zero (cold device), the components inside the dash box of the equivalent circuit can be ignored. According to the definition of $Y$-parameters and (1), the $16 Y$-parameters in (1) which represent the equivalent circuit in Fig. 1 can be obtained. However, six independent $Y$-parameters are sufficient to extract the components in the equivalent circuit for a cold device. Six independent $Y$-parameters including $Y_{\mathrm{GG}}, Y_{\mathrm{SG}}, Y_{\mathrm{DG}}, Y_{\mathrm{BS}}, Y_{\mathrm{BD}}$, and $Y_{\mathrm{SD}}$ were derived in terms of the components in the small signal equivalent circuit of a cold device. The higher order terms of $\left(\omega C_{\mathrm{x}} R_{\mathrm{x}}\right)$ were neglected because they are much smaller than $\omega C_{\mathrm{x}} R_{\mathrm{x}}$ and $\left(\omega C_{\mathrm{x}} R_{\mathrm{x}}\right)^{2}$ within the measurement frequency range. And the six $Y$-parameters can be approximated as

$$
\begin{aligned}
\operatorname{Im}\left[Y_{\mathrm{GG}}\right] \approx & j \omega\left(C_{\mathrm{gso}}+C_{\mathrm{gdo}}+C_{\mathrm{gbo}}\right) \\
\operatorname{Im}\left[Y_{\mathrm{SG}}\right] \approx & -j \omega C_{\mathrm{gso}} \\
\operatorname{Im}\left[Y_{\mathrm{DG}}\right] \approx & -j \omega C_{\mathrm{gdo}} \\
Y_{\mathrm{BS}} \approx & j \omega C_{s b o}+\omega^{2} C_{s b o}{ }^{2}\left(R_{\mathrm{sb}}+R_{\mathrm{bb}}\right) \\
& +\omega^{2} C_{s b o} C_{d b o} R_{\mathrm{bb}} \\
Y_{\mathrm{BD}} \approx & j \omega C_{d b o}+\omega^{2} C_{d b o}{ }^{2}\left(R_{\mathrm{db}}+R_{\mathrm{bb}}\right) \\
& +\omega^{2} C_{s b o} C_{d b o} R_{\mathrm{bb}} \\
\operatorname{Re}\left[Y_{\mathrm{SD}}\right] \approx & \omega^{2} C_{s b o} C_{d b o} R_{\mathrm{bb}} .
\end{aligned}
$$

The footnote "o" in $C_{\mathrm{gso}}, C_{\mathrm{gdo}}, C_{\mathrm{gbo}}, C_{s b o}$, and $C_{d b o}$ denotes the values of these components in a cold device. According to (2)-(4), $C_{\text {gso }}, C_{\text {gdo }}$ and $C_{\text {gbo }}$ can be directly 


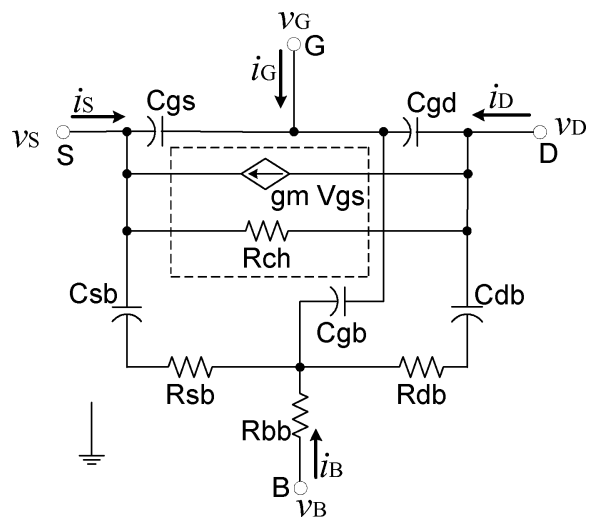

Fig. 1. Small-signal equivalent circuit of a four-port RF MOSFET. The components in dashed box are corresponded to the device operated in saturation region, and they are ignored while zero bias voltage is applied on each terminal.

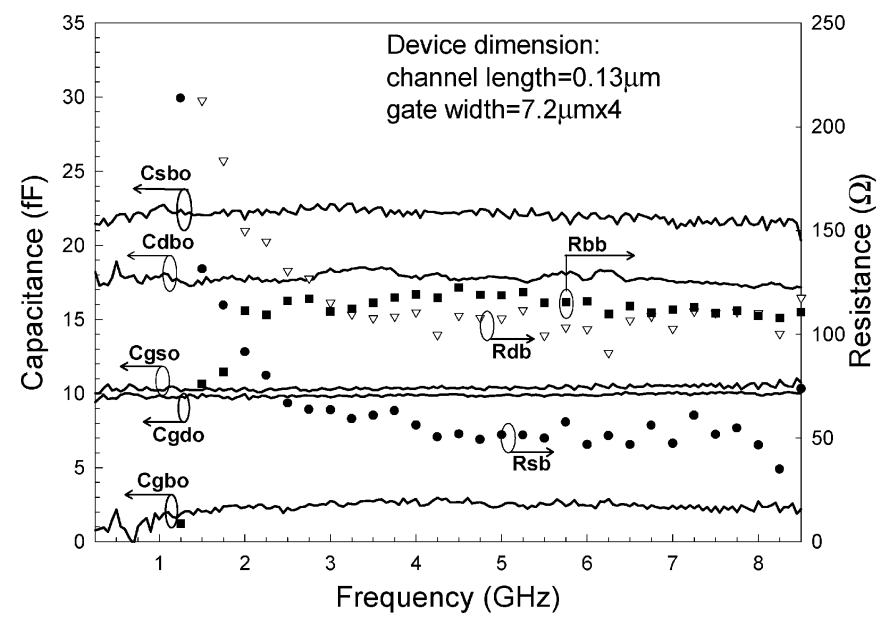

Fig. 2. Frequency dependence of $C_{\mathrm{gso}}, C_{\mathrm{gdo}}, C_{\mathrm{gb}}, C_{\mathrm{sb}}, C_{\mathrm{db}}, R_{\mathrm{sb}}, R_{\mathrm{db}}$, and $R_{\mathrm{bb}}$ of a cold device extracted from $Y$-parameters measured by four-port measurement.

extracted from the imaginary part of $Y_{\mathrm{SG}}, Y_{\mathrm{DG}}$ and $Y_{\mathrm{GG}}$. From $\operatorname{Im}\left[Y_{\mathrm{BS}}\right]$ and $\operatorname{Im}\left[Y_{\mathrm{BD}}\right], C_{s b o}$ and $C_{d b o}$ are also extracted. As the values of these five capacitances are known, $R_{\mathrm{bb}}$ can be obtained by (7), then $R_{\mathrm{sb}}$ and $R_{\mathrm{db}}$ can be extracted according to (5) and (6).

\section{RESULTS AND DISCUSSIONS}

Three 0.13- $\mu \mathrm{m}$ nMOSFETs with different gate widths were characterized on wafer by Agilent E5071B 4-port network analyzer and cascade microwave probes from $250 \mathrm{MHz}$ to $8.5 \mathrm{GHz}$. The gate widths of the devices are $3.6 \mu \mathrm{m} \times 4,7.2 \mu \mathrm{m} \times 4$, and $12 \mu \mathrm{m} \times 4$, respectively. A 16 -entry $4 \times 4 S$-parameter matrix was obtained and transformed to $4 \times 4 Y$-parameters. The parasitics of test pad were de-embedded by open and short dummy structures. Fig. 2 shows the extracted results of $C_{\mathrm{gso}}, C_{\mathrm{gdo}}$, $C_{\mathrm{gbo}}, C_{s b o}, C_{d b o}, R_{\mathrm{sb}}, R_{\mathrm{db}}$, and $R_{\mathrm{bb}}$ for the device with gate width of $7.2 * 4 \mu \mathrm{m}$. The values of the capacitances are almost constant among the measuring frequency. However, the extracted $R_{\mathrm{sb}}, R_{\mathrm{db}}$, and $R_{\mathrm{bb}}$ below $2.5 \mathrm{GHz}$ are irregular because the effect of substrate resistances on the output characteristics of RF MOSFETs is minor at lower frequency range.
TABLE I

PARAMETERS EXTRACTED FROM COLD DEVICES

\begin{tabular}{c|c|c|c|c|c|c|c|c}
\hline & $\begin{array}{c}\mathrm{C}_{\mathrm{gso}} \\
(\mathrm{fF})\end{array}$ & $\begin{array}{c}\mathrm{C}_{\mathrm{gdo}} \\
(\mathrm{fF})\end{array}$ & $\begin{array}{c}\mathrm{C}_{\mathrm{sbo}} \\
(\mathrm{fF})\end{array}$ & $\begin{array}{c}\mathrm{C}_{\mathrm{dbo}} \\
(\mathrm{fF})\end{array}$ & $\begin{array}{c}\mathrm{C}_{\mathrm{gbo}} \\
(\mathrm{fF})\end{array}$ & $\begin{array}{l}\mathrm{R}_{\mathrm{db}} \\
(\Omega)\end{array}$ & $\begin{array}{c}\mathrm{R}_{\mathrm{sb}} \\
(\Omega)\end{array}$ & $\begin{array}{c}\mathrm{R}_{\mathrm{bb}} \\
(\Omega)\end{array}$ \\
\hline $\begin{array}{c}\mathrm{M} 1 \\
3.6 \mu m \times 4\end{array}$ & 5.9 & 5.5 & 11.5 & 9.2 & 1.1 & 260 & 160 & 175 \\
\hline $\begin{array}{c}\mathrm{M} 2 \\
7.2 \mu m \times 4\end{array}$ & 10.3 & 9.8 & 22 & 18 & 2.5 & 100 & 50 & 115 \\
\hline $\begin{array}{c}\mathrm{M} 3 \\
12 \mu m \times 4\end{array}$ & 16.3 & 15.6 & 37 & 29.5 & 4.5 & 50 & 15 & 77 \\
\hline
\end{tabular}

The extracted parameters of three $0.13 \mu \mathrm{m}$ RF MOSFETs with different dimensions. The gate widths of these three devices are $3.6 \times 4 \mu \mathrm{m}, 7.2 \times 4 \mu \mathrm{m}, 12 \times 4 \mu \mathrm{m}$, respectively.

The real parts of $Y_{\mathrm{BD}}, Y_{\mathrm{BS}}$ and $Y_{\mathrm{BB}}$ are very small at frequency below $2.5 \mathrm{GHz}$, and will be limited by the measurement error. It makes the values of substrate resistances are valid from $2.5 \mathrm{GHz}$ to $8.5 \mathrm{GHz}$ in this study.

Table I lists the capacitances and resistances extracted from the three cold devices with different dimensions. Because of the even gate finger number, there is one more source junction than the drain junctions. These three devices are structurally unsymmetrical, the extracted values do tell this point. The scalability of these parameters with the device dimension is also observed.

The substrate resistances are unable to be extracted from a MOSFET biased in active region, because of the more significant intrinsic components $\left(g_{\mathrm{m}}, R_{\mathrm{ch}}\right)$ in active region. However, since the substrate resistances have weak dependence on the bias condition [1], the corresponding values extracted from a cold device can be used in an active device. To verify the extracted substrate resistances, the bias dependent components $g_{\mathrm{m}}, R_{\mathrm{ch}}$, $C_{\mathrm{gd}}, C_{\mathrm{gs}}, C_{\mathrm{db}}, C_{\mathrm{sb}}$, and $C_{\mathrm{gb}}$ in Fig. 1 were extracted from 4-port measurement data of the four-port device biased in active region $\left(\mathrm{V}_{g}=\mathrm{V}_{\mathrm{D}}=1 \mathrm{~V}, \mathrm{~V}_{S}=\mathrm{V}_{B}=0 \mathrm{~V}\right) . R_{\mathrm{ch}}$ was directly extracted from the real part of $Y_{\mathrm{DD}}$ at low frequency range while the substrate coupling effect is minor [6]. $g_{\mathrm{m}}$ was extracted from the real part of $Y_{\mathrm{DG}}$ at low frequency range [6]. And the $C_{\mathrm{gd}}, C_{\mathrm{gs}}, C_{\mathrm{db}}$ were extracted according to (2)-(4). $C_{\mathrm{sb}}$, and $C_{\mathrm{gb}}$ were extracted from the imaginary part of (5) and (6). The equivalent circuit in Fig. 1 incorporated with the substrate resistances extracted from cold devices and the bias dependent components extracted from active devices were simulated by ADS software. The simulation data of output admittance $Y_{\mathrm{DD}}$ were compared with the output admittance of common source RF MOSFETs measured by conventional two-port method. The results were shown in Fig. 3 and good agreement between the simulation and measurement data up to $26.5 \mathrm{GHz}$ was obtained. The modeling results of bias dependence of $Y_{\mathrm{DD}}$ are also shown in the inset of Fig. 3.

The validity of (5)-(7) were verified by comparing the fourport measurement data and the simulated data of the equivalent circuit of cold devices (Fig. 1 with $g_{\mathrm{m}}, R_{\mathrm{ch}}$ removed). The results of M3 devices were shown in Figs. 4 and 5. It indicates the assumption of neglected the higher order terms of $\left(\omega C_{\mathrm{x}} R_{\mathrm{x}}\right)$ is reasonable within the measurement frequency $(<8.5 \mathrm{GHz})$. Figs. 4 and 5 also demonstrate the results of the six $Y$-parameters of active M3 device. The differences between measurement and simulated data were also small. 


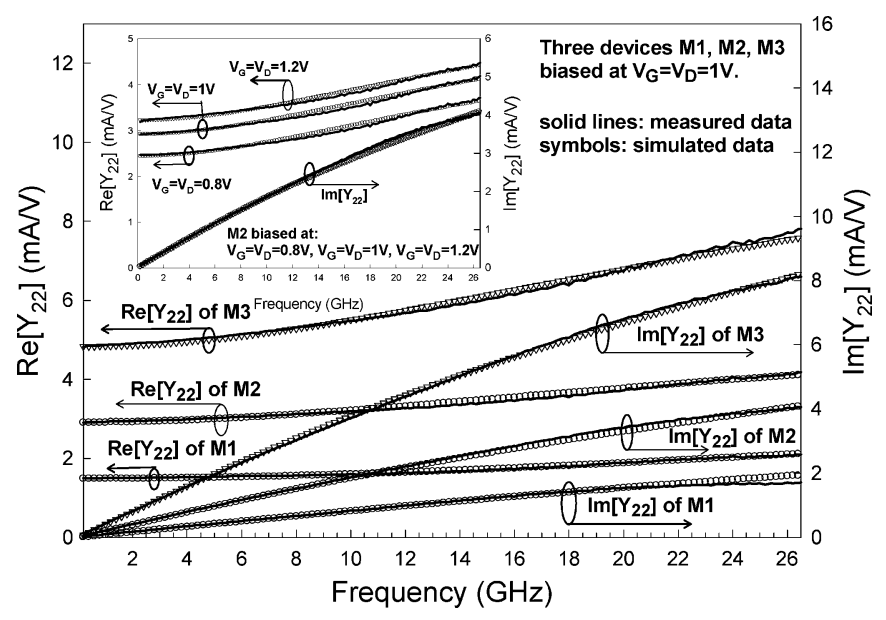

Fig. 3. Measured and simulated $Y_{22}$ of three different conventional two-port common-source RF MOSFETs biased at $\mathrm{V}_{g}=1 \mathrm{~V}, \mathrm{~V}_{\mathrm{D}}=1 \mathrm{~V}$. The two-port simulation is performed based on the proposed small-signal equivalent circuit in Fig. 1 with the source and substrate terminals grounded. The modeling results of the device M2 with different bias are also shown in the inset.

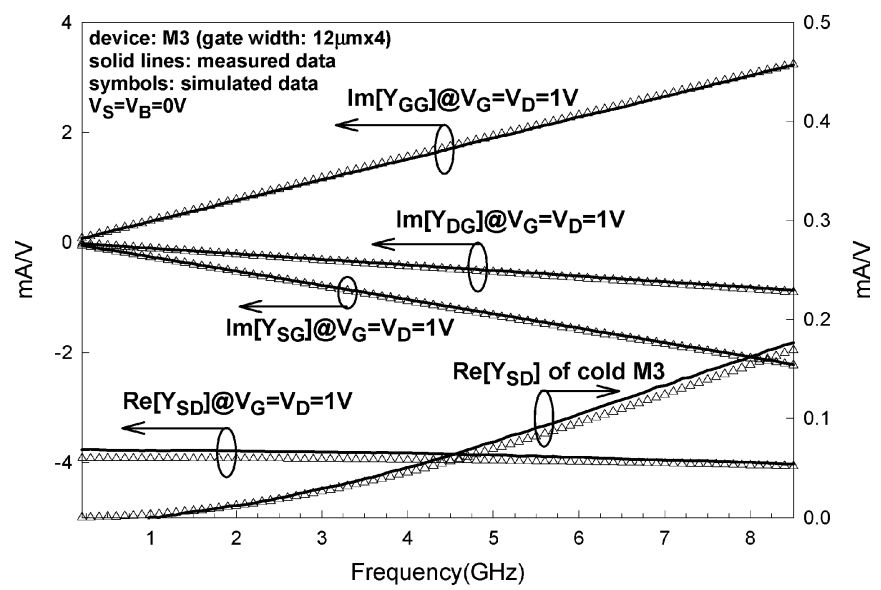

Fig. 4. Measured and simulated $\operatorname{Im}\left[Y_{\mathrm{GG}}\right], \operatorname{Im}\left[Y_{\mathrm{DG}}\right], \operatorname{Im}\left[Y_{\mathrm{SG}}\right], \operatorname{Re}\left[Y_{\mathrm{SD}}\right]$ of M3 device, the $\operatorname{Re}\left[Y_{\mathrm{SD}}\right]$ include both active and cold condition.

\section{CONCLUSION}

An extraction method for substrate resistances and extrinsic capacitances of RF MOSFETs based on four-port measurement are demonstrated for the first time. A T-liked substrate resistance network is proposed and the components are extracted from four-port measurement data directly and simply. The extracted values show scaling with the device dimension, and the proposed substrate network well models the substrate loss of RF

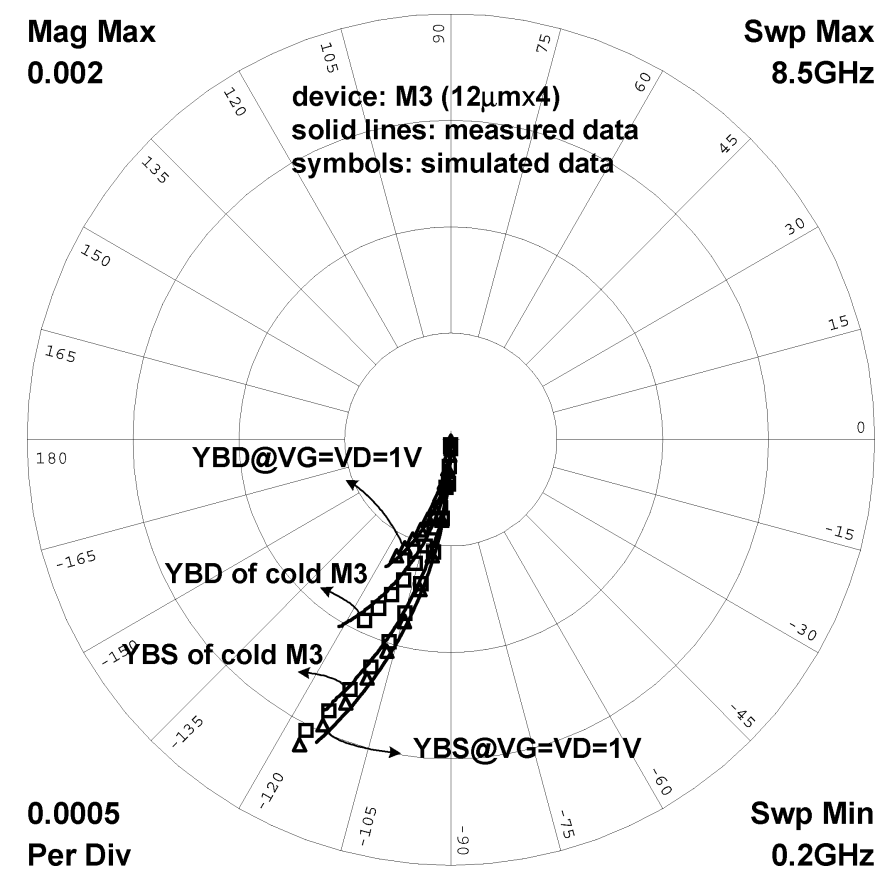

Fig. 5. Measured and simulated YBS and YBD of M3 device in both active and cold condition.

MOSFETs up to $26.5 \mathrm{GHz}$ for different dimensions and bias voltages.

\section{REFERENCES}

[1] J. Han, M. Je, and H. Shin, "A simple and accurate method for extracting substrate resistance of RF MOSFETs," IEEE Electron Device Lett., vol. 23, no. 7, pp. 434-436, Jul. 2002.

[2] M. Je and H. Shin, "Gate bias dependent of substrate signal coupling effect in RF MOSFETs," IEEE Electron Lett., vol. 24, no. 3, pp. 183-185, Mar. 2003.

[3] Y. Cheng and M. Matloubian, "On the high-frequency characteristics of substrate resistance in RF MOSFETs," IEEE Electron Lett., vol. 21, no. 12, pp. 604-606, Dec. 2000.

[4] _ - "Parameter extraction of accurate and scaleable substrate resistance components in RF MOSFETs," IEEE Electron Lett., vol. 23, no. 4, pp. 221-223, Apr. 2002.

[5] C.-H. Kim, C.-S. Kim, H.-K. Yu, and K.-S. Nam, "Unique extraction of substrate parameters of common-source MOSFETs," IEEE Microw. Guided Wave Lett., vol. 9, no. 3, pp. 108-110, Mar. 1999.

[6] S. H.-M. Jen, C. C. Enz, D. R. Pehlke, M. Schroter, and B. J. Sheu, "Accurate modeling and parameter extraction for MOS transistors valid up to $10 \mathrm{GHz}$," IEEE Tran. Electron Devices, vol. 46, no. 11, pp. 2217-2227, Nov. 1999.

[7] S. D. Wu, G. W. Huang, L. P. Cheng, and C. Y. Chang, "Characterization of 2-port configuration MOSFETs amplifiers by 4-port measurement,' in Proc. Asia-Pacific Microwave Conf., vol. 3, 2003, pp. 1431-1433. 